\title{
CARACTERÍSTICAS DEL CICLO BIOLÓGICO DE Lucilia sericata (MEIGEN, 1826) (DIPTERA: CALLIPHORIDAE) SOBRE DIETAS DIFERENTES
}

\section{CHARACTERISTICS OF THE BIOLOGICAL CYCLE OF Lucilia sericata (MEIGEN, 1826) (DIPTERA: CALLIPHORIDAE) ON DIFFERENTS DIETS}

\author{
Tatiana Pinilla B. ${ }^{1}$, Yisethe Acuña ${ }^{1}$, Diana Cortes B. ${ }^{1}$, Andrea Díaz R. ${ }^{2}$, Alexandra Segura ${ }^{3}$, Felio J. Bello ${ }^{4}$
}

\begin{abstract}
${ }^{1}$ Estudiante pregrado. Laboratorio de Entomología Médica y Forense, Facultad de Ciencias Naturales y Matemáticas, Universidad del Rosario ${ }^{2}$ Bióloga. Laboratorio de Entomología Médica y Forense, Facultad de Ciencias Naturales y Matemáticas, Universidad del Rosario ${ }^{3}$ M.Sc. Estudiante de Doctorado Ciencias Biomédicas. Laboratorio de Entomología Médica y Forense, Facultad de Ciencias Naturales y Matemática, Universidad del Rosario ${ }^{4}$ M.Sc. Ph.D. Laboratorio de Entomología Médica y Forense, Facultad de Ciencias Naturales y Matemática, Universidad del Rosario. Bogotá D.C., Colombia. Dirección de correspondencia: Calle 63D No. 24-31, Email: felio.bello@urosario.edu.co
\end{abstract}

Rev. U.D.C.A Act. \& Div. Cient. 13 (2): 153-161, 2010

\section{RESUMEN}

Lucilia sericata (Diptera: Calliphoridae) es una mosca necrófaga de importancia médica y forense, utilizada en terapia larval y su desarrollo biológico es usado para establecer el intervalo postmortem. Para criar esta especie, generalmente, se recurre a tejidos animales que al descomponerse producen malos olores y contaminación. El objetivo de este estudio fue determinar las características del ciclo biológico de $L$. sericata empleando cuatro dietas diferentes a base de hígado de res $(\mathrm{H})$, leche en polvo (LP), pescado (P) e hígado en polvo (HP). El estudio, se inició a partir de huevos tomados de una colonia de $L$. sericata, previamente establecida, bajo condiciones de laboratorio $\left(24-27^{\circ} \mathrm{C}, 60 \% \mathrm{HR}\right.$, fotoperiodo 12 horas luz). La dieta con la que se obtuvo la mayor duración en el ciclo de vida de $L$. sericata, desde huevo hasta pupa, fue LP y el menor tiempo de desarrollo de la especie, se estableció en la dieta HP. Por otro lado, la menor longevidad de las moscas, se consiguió en la dieta $\mathrm{H}$; sin embargo, en este sustrato alimenticio, los insectos alcanzaron un tamaño mayor. De manera general, se registró un porcentaje mayor de hembras en las dietas LP y P. Potencialmente, las tres dietas sintéticas evaluadas presentaron características de sustratos alimenticios, óptimos para la cría de la mosca, en condiciones de laboratorio.

Palabras clave: Moscas, Entomología Forense, dietas sintéticas, ciclo de vida, terapia larval, intervalo post-mortem.

\section{SUMMARY}

Lucilia sericata (Diptera: Calliphoridae) is a necrophagous fly of medical and forensic importance used in larval therapy, being its biological development important to establish the post postmortem interval. Animal tissues are generally employed to breed this specie, but these substrates, while decomposing produce bad odors and contamination. The aim of this study was to determine the characteristics of the life cycle of $L$. sericata using four different diets: beef liver $(\mathrm{H})$, milk powder (LP), fish (P) and liver powder (HP). This study started from eggs taken from a colony of $L$. sericata previously established under laboratory conditions $\left(24-27^{\circ} \mathrm{C}\right.$, $60 \% \mathrm{RH}$, photoperiod 12 hours light). The highest duration in the life cycle from egg to pupa was obtained on the diet LP, and the shortest developmental time was established on the diet HP. On the other hand the shortest longevity of adult flies was attained on the diet $\mathrm{H}$. However, in this food substrate the flies were larger than on the others diets. In general, a higher percentage of females was registered with diets LP and P. Potentially, the three synthetic diets evaluated, revealed characteristics of optimal substrates for the breeding of the flies under laboratory conditions.

Key words: Flies, Forensic Entomology, synthetic diets, life cycle, larval therapy, post-mortem interval. 


\section{INTRODUCCIÓN}

La mosca verde o Green Bottle Fly, Lucilia sericata (Meigen, 1826) (Diptera: Calliphoridae) es una mosca cosmopolita con un alto grado de sinantropía, es decir, en estrecha relación con los asentamientos humanos. Su distribución, se ha reportado en el neotrópico, en países como Argentina, Brasil, Chile y Perú. En Colombia, se ha registrado en los departamentos de Antioquia, Cundinamarca, Santander y Sucre (Pape et al. 2004).

L. sericata es de importancia para las ciencias forenses y médicas y está referenciada como una de las primeras especies colonizadoras que predomina en la fauna cadavérica (Figueroa E Linhares, 2002; Camacho, 2003), razón por la cual, se ha empleado para determinar el intervalo post-mortem (IPM) (Saigusa et al. 2009). Por otro lado, las larvas de esta mosca son eficaces en tratamientos de heridas crónicas de difícil cicatrización, ya que actúan como parásitos facultativos, alimentándose solo del tejido necrótico. La acción de las larvas sobre las heridas está científicamente comprobada, a través de tres funciones primordiales: desbridamiento, actividad antimicrobiana y estimulación del tejido de granulación (Horobin et al. 2005; Parnés E Lagan, 2007). En la actualidad, la terapia larval es considerada una tecnología natural, simple, segura y altamente exitosa para la curación de úlceras o lesiones crónicas en humanos y animales (Gentil \& Esmirnova, 2009; Sherman, 2002). Además, el uso de larvas para el desbridamiento es una alternativa rápida y de bajo costo, con la que se logra un tejido de granulación adecuado para la aplicación de equivalentes cutáneos autólogos, que constituyen una opción para pacientes refractarios a otros tratamiento (Escobar et al. 2007).

Para la cría en masa de $L$. sericata, bajo condiciones de laboratorio, generalmente, se emplean tejidos orgánicos que al descomponerse producen malos olores y contaminación, lo cual, hace necesario implementar dietas sintéticas que disminuyan los anteriores factores. De esta manera, no se presentan dificultades para el medio ambiente, ni para la salud de los investigadores, disponiéndose así de un óptimo sustrato para el desarrollo continuo del ciclo biológico de la especie. Así mismo, cuando se utilizan huevos de esta especie, ovipositados por hembras alimentadas con dietas artificiales y con menor carga de microorganismos en su ambiente, las larvas que eclosionan, previo tratamiento aséptico de la superficie de los huevos, estarán libres de agentes microbianos y podrán ser empleadas en terapia larval, con menos riesgos para la salud de los pacientes.

Según Tachibana E Numata (2001), Bogdanow fue pionero en la cría de insectos necrófagos al utilizar una dieta sintética, compuesta, principalmente, de extracto de carne y la especie cultivada fue Calliphora vomitoria (Linnaeus, 1758)
(Diptera: Calliphoridae); a partir de entonces, pero de manera esporádica, la crianza de insectos con dietas artificiales, se ha destinado para llevar a cabo estudios experimentales en dípteros de la familia Calliphoridae. Las dietas artificiales para insectos, se clasifican en holídicas, que contienen elementos químicos puros; merídicas, con elementos químicamente conocidos y componentes químicamente no definidos y oligídicas, de material natural crudo, de origen animal o vegetal (Cibrián, 1994; Acatitla et. al. 2004).

Varios autores han manipulado diferentes sustratos en las dietas sintéticas, con el fin de identificar los que son vitales y más significativos para el desarrollo y crecimiento de los insectos, en condiciones controladas de laboratorio. Tal es el caso de la especie Calliphora stygia (Fabricius, 1781), que se crió en una dieta basada en aceite de oliva y levadura de vino (Ujvari et al. 2009). Chrysomya megacephala (Fabricius, 1794), se cultivó en una dieta con diferentes concentraciones de albúmina (Mendoca et al. 2009), mientras que Chrysomya albiceps (Wiedemann, 1819) fue criada para evaluar el desarrollo post-embrionario, empleando dietas artificiales, preparadas con diferentes tejidos animales (Estrada et al. 2009) y Cochliomyia hominivorax (Coquerel, 1858), se logró desarrollar en una dieta basada en carne, baja en grasa, con un sustrato de poliéster (Silva et al. 2008).

Para la cría de $L$. sericata se han evaluado varias dietas, las cuales, en mayor o menor grado, han sido óptimas para el desarrollo y el mantenimiento del ciclo de vida de la mosca; así, por ejemplo, Ring (1972) estableció una dieta compuesta de extracto de levadura y de leche entera, con resultados satisfactorios. De manera similar, Tachibana \& Numata (2001), con base a germen de trigo, leche en polvo y levadura seca lograron significativos avances en la cría del insecto. A su vez, Daniels \& Simkiss (1991) obtuvieron el desarrollo de la especie, a partir de una dieta con $20 \%$ de sangre de caballo y de levadura.

El objetivo del presente estudio fue determinar la duración del ciclo de vida, el tamaño de los insectos, la relación por sexos y la longevidad de $L$. sericata en cuatro dietas, tres de ellas sintéticas, con el fin de establecer un sustrato alimenticio eficaz para el desarrollo biológico y el mantenimiento de la especie en condiciones de laboratorio.

\section{MATERIALES Y MÉTODOS}

Preparación y composición de las dietas: Se emplearon tres sustratos como base proteica de dietas artificiales merídicas: leche en polvo (LP), hígado en polvo (HP) y alimento para pescado (P); la cuarta dieta consistió del sustrato natural de hígado de bovino $(\mathrm{H})$, cada una de ellas tuvo su respectiva 
réplica. La dieta de HP, descrita por Gingrich et al. (1971) fue modificada sustituyendo $6 \mathrm{~g}$ de hígado en polvo por $3 \mathrm{~g}$ de Incros (alimento para peces), obteniéndose, de esta forma, la dieta P. En la tabla 1, se muestra la composición de cada una de las dietas artificiales.

Insectos: El ciclo de vida de $L$. sericata, se inició a partir de una postura aproximada de 1300 huevos, la que se obtuvo de una colonia previamente establecida en el laboratorio de entomología de la Universidad del Rosario. Este material biológico, se dividió en ocho partes iguales, que se pesaron hasta alcanzar cada una de ellas 0,0260g (160 huevos, aproximadamente). Posteriormente, las masas de huevos fraccionadas, se colocaron, de forma individual, en ocho frascos numerados y marcados con los datos correspondientes de cada dieta: HP, H, LP y P, sustratos fueron utilizados para el desarrollo del ciclo biológico desde larva hasta el estado de pupa. Una vez emergidas las moscas adultas, se mantuvieron en jaulas, separadas, según la procedencia de las dietas, a temperatura de $24-27^{\circ} \mathrm{C}$, con humedad relativa de $60 \%$, fotoperiodo 12 horas luz y agua con azúcar al $40 \%$, como fuente de carbohidratos.

Datos del desarrollo biológico de L. sericata: La eficacia de cada dieta, se analizó teniendo en cuenta la duración en días de la especie para cada estado y estadio, el tamaño de los adultos (mm), la relación de sexos y la longevidad de los adultos. Para evaluar las anteriores variables, se emplearon protocolos donde se registraron, diariamente, los datos correspondientes.

Diez especímenes larvales fueron extraídos diariamente de cada uno de los frascos de cultivo de las diferentes dietas, para observar los espiráculos larvales, con el fin de establecer el estadio de desarrollo (larva I, larva II y larva III), con ayuda de un estereoscopio marca Leica S6D, para las observaciones respectivas. De igual manera, se efectuaron registros diarios para el estado de pupa.

Empleando un estereomicroscópio marca Nikon, modelo SMZ1500, compuesto por cámara digital de alta resolución, modelo DS-Ri1-U2 y software "NIS ELEMENT" para análisis de imagen, se midió la longitud del ala, desde la basicosta hasta la vena media costal, como estimador del tamaño de los adultos (Rodriguero et al. 2002)

Así mismo, se estableció la relación de sexos en cada dieta. Finalmente, la mortalidad de los adultos, se registró por conteo para cada uno de los cuatro sustratos alimenticios.

Análisis estadísticos: Los datos obtenidos a partir del ciclo biológico de la especie y su relación con las dietas utilizadas,

Tabla 1. Composición de las dietas artificiales usadas para comparar el ciclo de vida de L. sericata.

\begin{tabular}{|c|c|c|c|}
\hline INGREDIENTES & $\begin{array}{l}\text { HÍGADO EN POLVO } \\
\text { (Gingrich et al. 1971) }\end{array}$ & $\begin{array}{l}\text { ALIMENTO EN PESCADO } \\
\text { (Mod. Gingrich et al. 1971) }\end{array}$ & $\begin{array}{l}\text { LECHE EN POLVO } \\
\text { (Tachibana \& Numata, } \\
\text { 2001) }\end{array}$ \\
\hline $\mathrm{Na}_{2} \mathrm{HPO}_{4} 12 \mathrm{H}_{2} \mathrm{O}$ & $0,012 \mathrm{~g}$ & $0,012 \mathrm{~g}$ & ------- \\
\hline $\mathrm{KH}_{2} \mathrm{PO}_{4}$ & $0,012 \mathrm{~g}$ & $0,012 \mathrm{~g}$ & ------ \\
\hline $\mathrm{NaCl}$ & $1,2 \mathrm{~g}$ & $1,2 \mathrm{~g}$ & 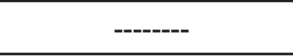 \\
\hline Hígado en polvo* & $6 \mathrm{~g}$ & ------- & ------- \\
\hline Alimento para peces & ---- & $3 \mathrm{~g}$ & ----- \\
\hline Glucosa & $1,2 \mathrm{~g}$ & $1,2 \mathrm{~g}$ & -.---- \\
\hline Agar nutritivo & $4,6 \mathrm{~g}$ & $4,6 \mathrm{~g}$ & -.--- \\
\hline $\mathrm{BHI}$ & $4,2 \mathrm{~g}$ & $4,2 \mathrm{~g}$ & 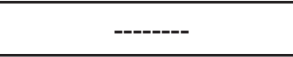 \\
\hline Sangre & $25 \mathrm{ml}$ & $25 \mathrm{ml}$ & ----- \\
\hline Leche entera en polvo & ------ & ------ & $12,5 \mathrm{~g}$ \\
\hline Levadura seca & ----- & ------ & $12,5 \mathrm{~g}$ \\
\hline Ácido acético & -.--- & ------ & $1,25 \mathrm{ml}$ \\
\hline Agar & - & 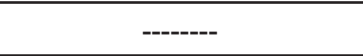 & $3,5 \mathrm{~g}$ \\
\hline Germen de trigo & ----- & ------- & $12,5 \mathrm{~g}$ \\
\hline Agua destilada & $200 \mathrm{ml}$ & $200 \mathrm{ml}$ & $250 \mathrm{ml}$ \\
\hline
\end{tabular}


se analizaron, en principio, con parámetros de estadística descriptiva, empleando las siguientes variables: tiempo de desarrollo, número de la muestra, sexo y tamaño corporal de $L$. sericata. De igual manera, la significancia de los datos obtenidos, a excepción del tamaño corporal, se evaluó con base en las pruebas de ANOVA, Fisher's con IC $=95 \%$ y con un $\mathrm{P}>0.05$, aplicando la corrección Bonferroni y, finalmente, el test $T$, con un IC $=99 \%$ y $\mathrm{P}>0.008$, utilizando, para tales efectos, los programas estadísticos Analysis of Variance Calculator-One-Way ANOVA from Summary Data (Soper, 2010) y Simple Interactive Statistical Analysis (http:// www.quantitativeskills.com/sisa/). Por otro lado, la prueba de Kruskal Wallis ( $\mathrm{gl}=3, \alpha=0,05, \mathrm{z}=2,6)$, se dispuso para establecer si hubo o no diferencias significativas en el tamaño de los dípteros.

\section{RESULTADOS Y DISCUSIÓN}

Duración del ciclo de vida de L. sericata en las cuatro dietas: Las dietas utilizadas en este estudio suministraron los nutrientes necesarios para que la totalidad del ciclo de vida de $L$. sericata tuviera lugar; sin embargo, se pudo establecer que entre las muestras del insecto de todas las dietas, hubo diferencias estadísticamente significativas en el tiempo de desarrollo ( $\mathrm{F}=242.260, \mathrm{P}<0,0001)$. De igual manera, los análisis realizados para esta misma variable entre pares de dietas: $\mathrm{H}$ y LP $(t=18.9, \mathrm{P}<0,0001) ; \mathrm{H}$ y HP $(t=15.4, \mathrm{P}$ $<0,0001)$; H y P $(t=8.22, \mathrm{P}<0,0001)$; LP y HP $(t=-2.9$, $\mathrm{P}$ $<0.0039)$; LP y $\mathrm{P}(t=-10.9, \mathrm{P}<0,0001)$ y $\mathrm{P}$ y $\mathrm{HP}(t=7.7$,
$\mathrm{P}<0,0001)$, también presentaron diferencias significativas, lo que concuerda con estudios previos de otras especies de la misma familia, siendo la dieta LP la que mostró mayor duración en el ciclo de vida de $L$. sericata, con un valor de $38 \pm 1.41$ días; contrariamente, en la dieta HP, se registró el menor tiempo de desarrollo de 26 días (Figura 1), lo que concuerda con el estudio realizado por Usaquén y Camacho (2004), donde el ciclo de vida de esta especie, en un sustrato natural, tuvo una duración de 35 días.

La duración del estado de huevo, el primer estadio larval y el segundo estadio larval fue de un día para todos los insectos, en las cuatro dietas, a diferencia de lo reportado en un estudio realizado con $L$. eximia, en donde el estado de huevo duró quince horas (Vélez et. al. 2008). El estadio larval III reflejó una duración de 6,7 días en la dieta $\mathrm{H}, 8$ días en la dieta HP y en las dietas de LP y P, se registró una duración de 8,5 y 9 días, respectivamente; no obstante, la duración del estado LIII, pupa y adulto fue un poco más larga en la dieta de LP que en las demás dietas, permitiendo que los adultos vivan más tiempo $\mathrm{y}$, consecuentemente, puedan generar más descendencia. Por otro lado, al utilizarse una dieta relativamente baja en grasa, como la dieta LP, aumenta la longevidad de las moscas (Zhang et al. 2009).

Para las dietas $\mathrm{HyHP}$ se presentaron dos estados en el mismo intervalo de tiempo (pupa y adulto), entre los días 16 - 23 y $14-23$, respectivamente; de igual forma, con las dietas LP y $\mathrm{P}$, en donde se manifestaron los estados de LIII, pupa y adulto de forma simultánea, entre los días 16 - 20 y 15 - 25,

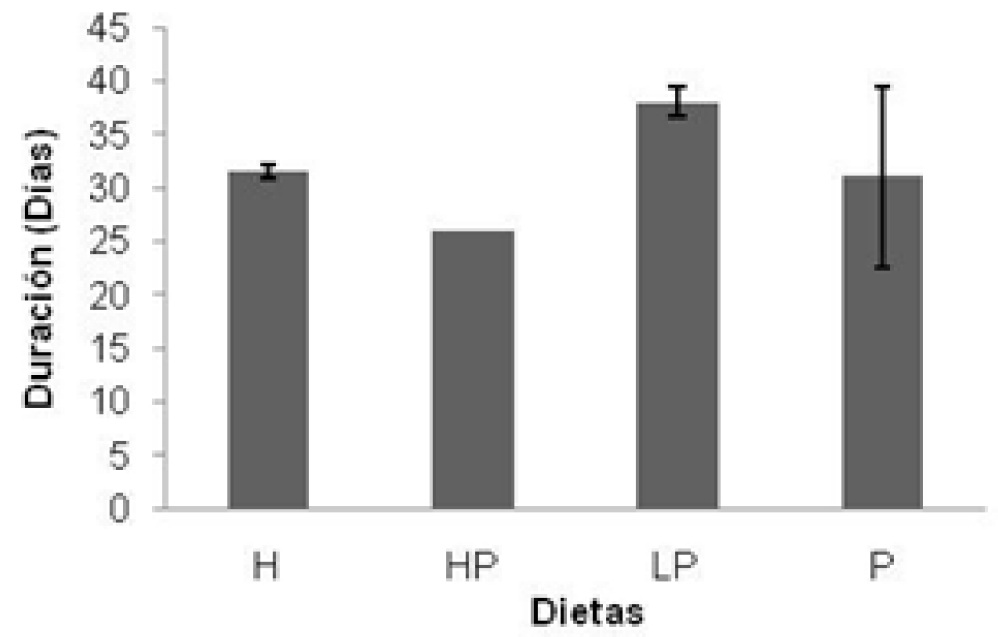

Figura 1. Duración del ciclo de vida Lucilia sericata y desviación estándar en las cuatro dietas: Hígado (H) 31,5 $\pm 0,71$; Hígado en polvo (HP) 26; Leche en Polvo (LP) 38 $\pm 1,41$ y Pescado (P) 31 $\pm 8,48$. 
respectivamente. La duración del estado adulto fue más larga en la dieta LP y, la más corta, en la dieta HP, con una duración de 14 y 8 días, respectivamente. A pesar que la dieta $\mathrm{H}$ no fue el mejor sustrato alimenticio en nuestro estudio, debido a que la muerte de los individuos fue más temprana, se han reportado otros trabajos que demuestran una mayor duración en el ciclo de vida y menor mortalidad de los individuos en dietas naturales; así, por ejemplo, Mendoca et al. (2009), en experimentos con individuos de la especie Crysomya megacephala, compararon el desarrollo biológico de la mosca en diferentes dietas sintéticas y carne bovina descompuesta, siendo esta última la que expuso mayor efectividad. Tenquist (1971) y Saunders et al. (1986) criaron larvas de L. sericata sobre dieta artificial, basada en leche en polvo y levadura seca, sustratos no fueron considerados adecuadas en los experimentos.

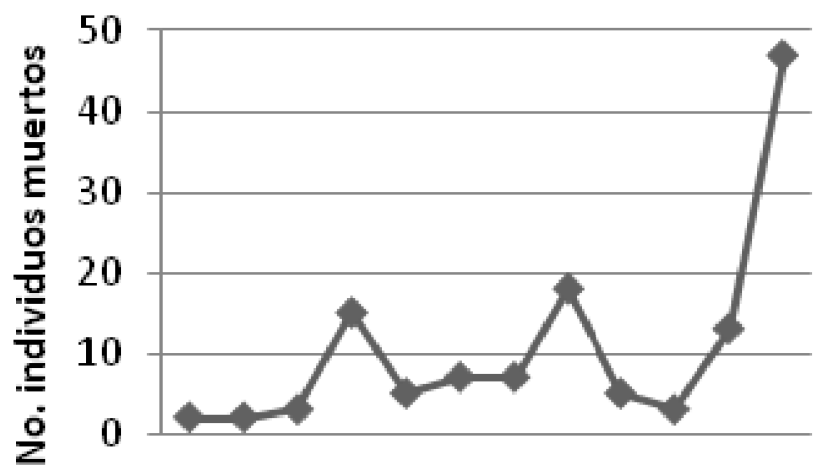

192425293031323334353640 Días $\quad$-LP

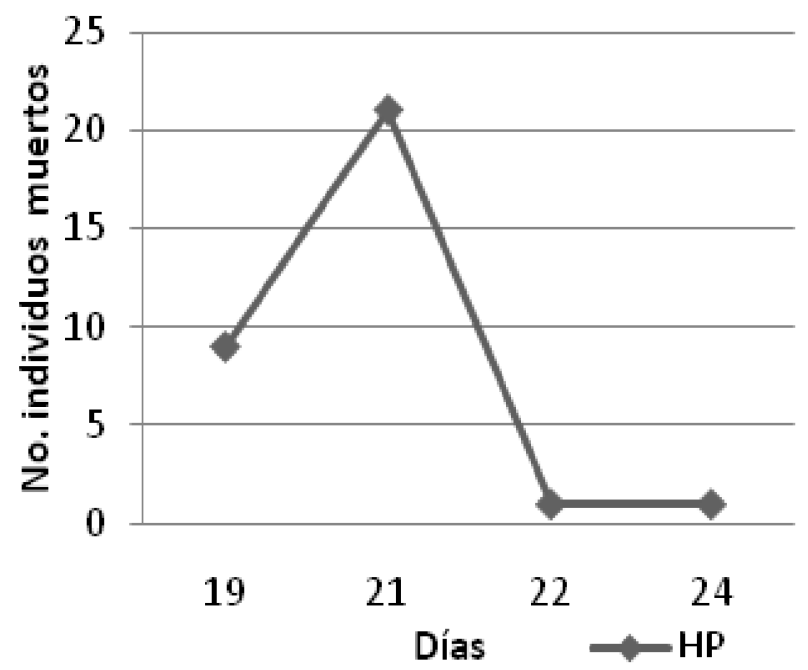

Longevidad de los adultos por cada dieta: En la mayoría de las dietas, se presentó la emergencia de los primeros adultos, entre el día 14 y 15, a excepción de la dieta P, cuyos individuos iniciaron esta etapa desde el día 22 (Figura 2). En cada una de las dietas hubo diferencias significativas en la longevidad de los adultos ( $P<0,0001 ; F=101,43)$. La mayor longevidad, se registró en LP, debido a que los individuos permanecieron hasta el día 38, a diferencia de la dieta HP, en donde los adultos sólo duraron hasta el día 26 existiendo, de esta manera, diferencias significativas $(P<0,00001 ; t=34,13)$. A pesar que las tres dietas sintéticas y el hígado natural demostraron ser sustratos alimenticios indispensables para el crecimiento de L. sericata, se destaca la dieta LP como la óptima, debido a que el colesterol de la leche, el ergosterol en la levadura y el sitosterol del germen de trigo, presentes en esta dieta, han sido factores nutritivos clave en el crecimiento y en el
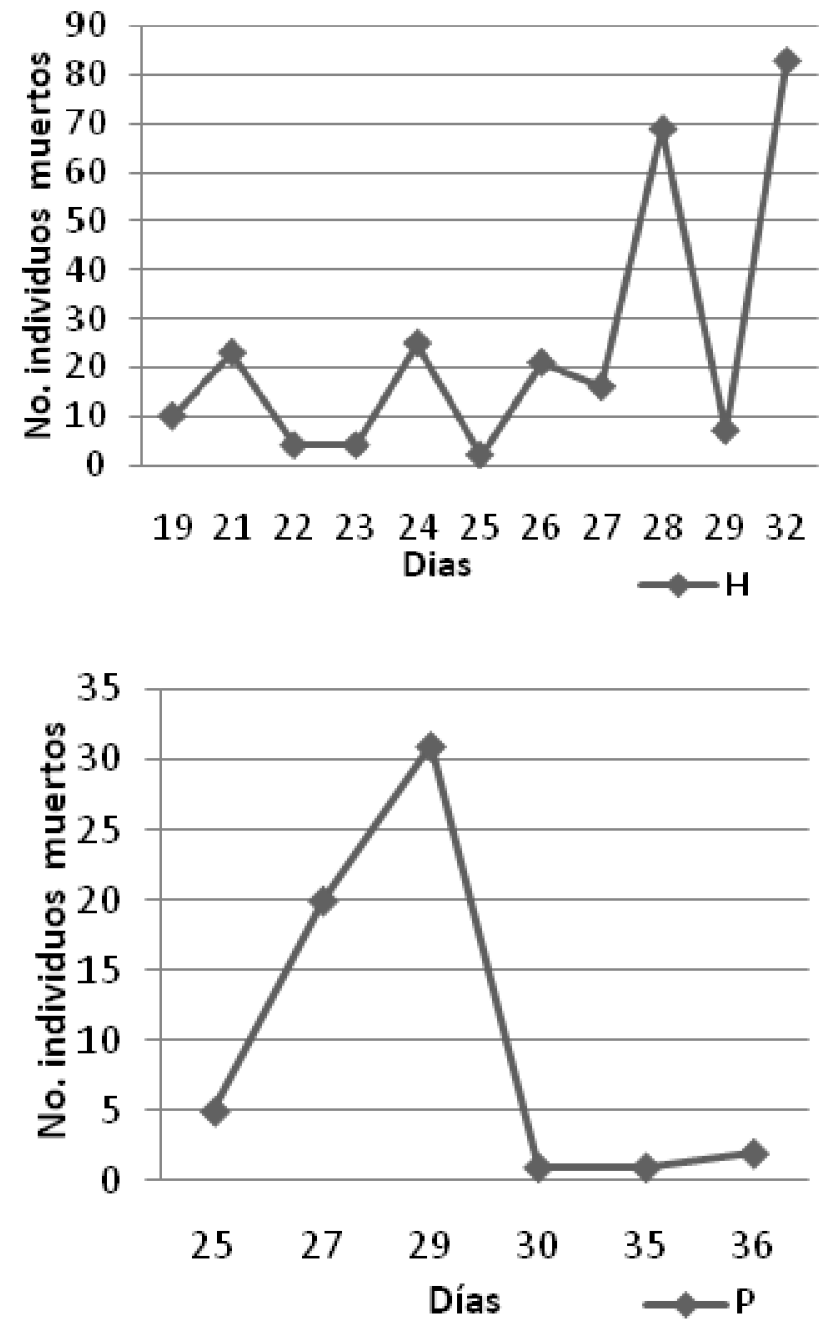

Figura 2. Supervivencia de los individuos de L. sericata por cada una de las dietas. Leche en Polvo (LP); Hígado (H); Hígado en polvo (HP) y Alimento para Pescado (P) 
desarrollo larval de varias especies de dípteros (Tachibana $\&$ Numata, 2001).

Adicionalmente, las hembras vivieron más tiempo que los machos (Figura 3), existiendo diferencias significativas entre la supervivencia de machos y de hembras $(P=0,0001$; $\mathrm{F}=29,73$ ), ya que su función fundamental radica en la acción de reproducirse y, por lo tanto, deben vivir el tiempo suficiente para cumplir esta meta y ovipositar el mayor número de huevos (Carles, 1997).

El colesterol es el principal esterol encontrado en los insectos, que sirve como un componente estructural de las membranas celulares y actúa como precursor de ecdisteroides, siendo fundamental en el desarrollo normal de la mayoría de los insectos (Zhang et al. 2009), por lo tanto, la efectividad de la dieta LP, se debe a que es considerada como una fuente rica en colesterol. También, otros estudios han demostrado que la dieta LP es una de las más manipuladas para el desarrollo de los insectos, debido a su composición en dos importantes proteínas: caseina y lactoalbumina (Loureiro et al. 2005). No obstante, aunque la dieta $\mathrm{P}$ no tuvo la misma relevancia que la LP en el presente trabajo, se ha podido establecer que en otros estudios esta dieta ha sido considerada como un sustrato preferencial para la oviposición de las moscas y, secundariamente, para la obtención de la fuente de proteínas (Carrero et al. 2004).

Tamaño de los dípteros por cada dieta: La tabla 2 muestra la longitud del ala de los dípteros según el tipo de dieta, discriminando entre hembras y machos. Los resultados obtenidos de la comparación estadística entre la dieta $\mathrm{H}$ con las demas dietas, mostró diferencias significativas para HP $(\breve{\mathrm{R}}=42,54>\mathrm{K}=39,18)$, LP $(\breve{\mathrm{R}}=180,05>\mathrm{K}=67,92)$ y $\mathrm{P}$ $(\breve{R}=104,38>K=51,90)$. Las moscas de mayor tamaño, se encontraron en la dieta $\mathrm{H}$, a diferencia de la dieta $\mathrm{HP}$, que presentó las menores mediciones. La dieta LP fue la segunda en orden de importancia, donde se exteriorizó el tamaño mayor para los adultos, existiendo diferencias significativas al compararla con $\mathrm{HP}(\check{\mathrm{R}}=137,51>\mathrm{K}=71,77)$, contrario a los resultados obtenidos sobre esta variable en la dieta $P$, que no mostró diferencias significativas $(\breve{\mathrm{R}}=75,67>\mathrm{K}=79,43)$. El análisis de varianza no registró diferencias estadisticamente significativas entre los sexos, ni tampoco en el tamaño de los adultos en las dietas (ANOVA, $F=31,03 ; P=0,000 ; P>0,05 ; I C=95 \%$; sin embargo, se observó que las hembras en todas las dietas fueron mas grandes que los machos, posiblemente, determinado por la selección natural que ha incidido, a través de muchos años, en la evolución del tamaño, otorgando ventajas a unos u otros individuos del mismo sexo (García, 1999). En razón a lo anterior, las dietas de LP y P al presentar un mayor número de hembras que de machos, posibilitan la continuidad de la colonia al aumentar el número de oviposiciones.

Aunque la dieta $\mathrm{H}$ no fue la más óptima, se pudo establecer que los individuos, tanto de las formas inmaduras como de los adultos, tuvieron tamaños relativamente mayores, comparada con la dieta LP. Según Lopes (1941), citado por Loureiro et al. (2005), esto es resultado de la calidad y de la cantidad de alimento ingerido, el cual, influencia el desarrollo de los ovarios y la fecundidad de los adultos, obteniendo individuos de mayor tamaño. Esta situación, se puede deber a la disponibilidad de los nutrientes presentes en el sustrato alimenticio, a su rápida digestión y a las cantidades mayores de calorías, incluidas las grasas, que están presentes en dicha dieta. Además, el proceso de adaptación a los nutrientes es más fácil, si se tiene en cuenta que en su hábitat natural este sustrato es el utilizado para la alimentación. Loureiro et al. (2005) obtuvieron los mejores resultados con individuos del género Pattonella (Sarcophagidae), indicando que en la dieta control, a base de carne, se obtuvo la mayor efectividad, debido a que los dípteros se encontraban en condiciones y en sustratos naturales. Con todo, es probable que este hecho induzca una menor duración del ciclo de vida y una menor longevidad de los adultos, como consecuencia del acelerado proceso metabólico, en las condiciones ambientales a que fueron sometidos los insectos, en condiciones de laboratorio.

Tabla 2. Longitud del ala de la especie L. sericata en machos y en hembras por cada una de las dietas.

\begin{tabular}{|c|c|c|c|c|c|c|c|c|c|c|}
\hline \multirow[t]{2}{*}{ DIETAS } & \multicolumn{2}{|c|}{$\begin{array}{l}\text { Promedio } \\
\text { Longitud Ala }\end{array}$} & \multirow{2}{*}{$\begin{array}{c}\text { Max } \\
\text { Hembra }\end{array}$} & \multirow{2}{*}{$\begin{array}{c}\text { Min } \\
\text { Hembra }\end{array}$} & \multirow{2}{*}{$\begin{array}{c}\text { Max } \\
\text { Macho }\end{array}$} & \multirow[t]{2}{*}{ Min Macho } & \multirow[t]{2}{*}{ Ds hembra } & \multirow{2}{*}{$\begin{array}{c}\text { Ds } \\
\text { macho }\end{array}$} & \multirow{2}{*}{$\begin{array}{l}\text { \# Ind } \\
\text { Hembra }\end{array}$} & \multirow{2}{*}{$\begin{array}{l}\text { \# Ind } \\
\text { Macho }\end{array}$} \\
\hline & Hembra & Macho & & & & & & & & \\
\hline $\mathrm{H}$ & 5,53 & 5,28 & 6,36 & 4,23 & 5,95 & 4,29 & 0,24 & 0,17 & 2462 & 1507 \\
\hline $\mathrm{HP}$ & 4,81 & 4,65 & 5,71 & 4,42 & 5,72 & 3,85 & 0 & 0,07 & 35 & 131 \\
\hline LP & 5,29 & 5,14 & 6,18 & 3,99 & 6,05 & 3,91 & 0,34 & 0,41 & 548 & 442 \\
\hline$P$ & 5,19 & 4,76 & 5,84 & 4,31 & 5,64 & 3,01 & 0,24 & 0,05 & 182 & 298 \\
\hline
\end{tabular}




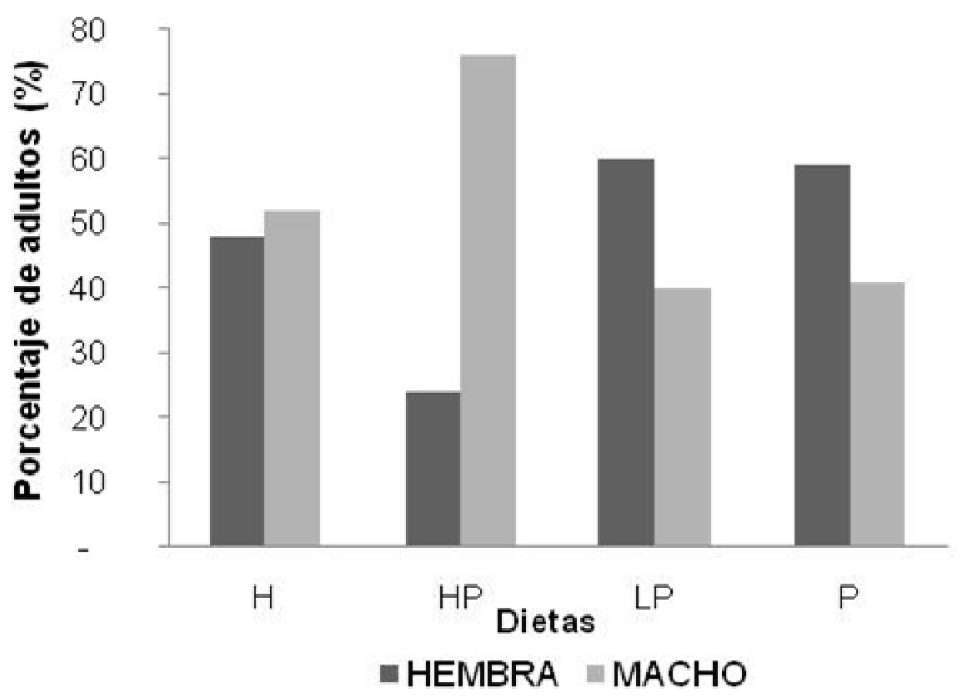

Figura 3. Porcentaje de machos y de hembras de L. sericata, en las diferentes dietas sintéticas.

Porcentaje de relación en sexos (hembras y machos) por dieta: La figura 3 describe el porcentaje en sexos obtenidos en cada una de las dietas. Los datos, se analizaron de forma cualitativa; se registró mayor cantidad de hembras para las dietas LP $(60,34 \%)$ y $P(58,93 \%)$, a diferencia de lo encontrado en las dietas HP (75,86\%) y H (52,16\%), que reflejaron una mayor proporción de machos que de hembras, aunque esta situación pude ser algo fortuito, determinada por otras causas, pues no se han encontrado información en la literatura que posibilite inferir la influencia de una dieta en particular, que sesgue la proporción del sexo de los insectos en una población.

Los sustratos alimenticios evaluados no revelaron contaminación alguna en el laboratorio, permitiendo así el suministro continuo de nutrientes a los insectos, lo que garantiza su mantenimiento para diferentes y posteriores estudios; sin embargo y teniendo en cuenta la importancia que representa la especie en la determinación del PMI, las dietas sintéticas no son útiles para determinar un ciclo de vida que sirva como base para establecer el tiempo de muerte de un individuo, ya que los nutrientes y las condiciones presentes en el ambiente natural varían en gran manera a los de las dietas artificiales, obteniéndose con éstas, una mayor duración en el ciclo de vida, en comparación con los sustratos naturales. Por otro lado, para la aplicación de terapia larval, Rey et al. (2008) demostraron que los especímenes criados en dietas artificiales cumplen los requerimientos para estos procedimientos y las larvas son efectivas en los proceso de remoción de tejido necrótico y cicatrización de las heridas crónicas, además, ofrecen mejores condiciones para la asepsia de los huevos, por la menor contaminación, como paso previo a la eclosión de las larvas estériles, que se aplican en bio-cirugía.

En conclusión, este estudio demostró que las dietas sintéticas utilizadas proporcionaron sustratos adecuados para la cría de $L$. sericata en condiciones de laboratorio, presentando diferencias significativas entre ellas, en concordancia con las variables analizadas, todas útiles para establecer colonias estables y en continuo crecimiento; no obstante, teniendo en cuenta las diferencias biológicas de $L$. sericata en las tres dietas sintéticas e Hígado, se establece la dieta LP como la más óptima para el desarrollo de la especie, al registrar una mayor duración en su ciclo de vida y manifestar individuos con mayores tamaños.

AGRADECIMIENTOS: A la Universidad del Rosario, por la financiación del presente trabajo. Conflictos de intereses: $\mathrm{El}$ manuscrito fue preparado y revisado con la participación de todos los autores, quienes declaramos que no existe ningún conflicto de intereses que ponga en riesgo la validez de los resultados presentados.

\section{BIBLIOGRAFÍA}

1. ACATITLA, C.; BAUTISTA, N.; VERA, J.; ROMERO, J.; CALYECAS, H. 2004. Ciclo biológico y tasas de supervivencia y reproducción de Copitarsia incommoda Walker (Lepidoptera: Noctuidae) en cinco dietas artificiales diferentes. Agrociencia. 38(03):355-363. 
2. CAMACHO, G. 2003. Sucesión de la entomofauna cadavérica y ciclo vital de Calliphora vicina (Diptera: Calliphoridae), como primera especie colonizadora, utilizando cerdo blanco (Sus scrofa) en Bogotá. Rev. Colomb. Entomol. 31(2):189-127.

3. CARRERO, V.; RODRIGUEZ, A.; AZEVEDO, A. 2004. Descrição quantitativa de Phaenicia eximia (Wiedemann, 1819) (Diptera, Calliphoridae) no campus da Universidade Federal Rural do Rio de Janeiro e sua atração por isca a base de sardinha: Estudo preliminar. Rev. Bras. Zoociências Juiz de Fora. 6(1):115-127.

4. CARLES, M. 1997. Los dípteros y el hombre. Barcelona Bol. S.E.A. 20:405-425.

5. CIBRIÁN, T.1994. Factores que influyen en la cría de insectos en condiciones controladas. En: Técnicas para la Cría de Insectos. Colegio de posgraduados. Instituto de Fitosanidad. p.11-23.

6. DANIELS, S.; SIMKISS, R. 1991. A simple larval diet for population studies on the blowfly Lucilia sericata (Diptera: Calliphoridae). Med. Vet. Entomol. 5:283-292.

7. ESCOBAR, M.; HENAO. J.; WOLFF, M.; ESTRADA, S.; RESTREPO, L. 2007. Tratamiento de las ulceras crónicas en los miembros inferiores con un equivalente cutáneo autologo y desbridacion con larvas de Lucilia sp. (Diptera: Calliphoridae). Reporte de un caso. Iatreia 20(4):397-406.

8. ESTRADA, D.; GRELLA, M.; THYSSEN, P.; LINHARES, A. 2009. Taxa de desenvolvimento de Chrysomya albiceps (Wiedemann) (Diptera: Calliphoridae) em dieta artificial acrescida de tecido animal para uso forense. Neotrop. Entomol. 38(2):203-207.

9. FIGUEROA, L.; LINHARES, A. 2002. Sinantropía de los Calliphoridae (Diptera) de Valdivia. Chile. Neotrop. Entomol. 31(2):233-239.

10. GARCÍA, E. 1999. Implicaciones ecológicas y evolutivas del tamaño en los artrópodos. Bol. S.E.A. 26:657-678.

11. GENTIL, I.; ESMIRNOVA, P. 2009. Larvaterapia. Revisión sistemática de evidencia científica. Rev. Internal de Ciencias Podológicas. 3(1):45-52.

12. GINGRICH, R.; GRAHAM, A.; HIGHTOWER, B. 1971. Media containing liquefied nutrients for mass-rearing larvae of the screwworm. J. Econ. Entomol. 64(3): 678-683.
13. HOROBIN, A.; SHAKESHEFF, K.; PRITCHARD, D. 2005. Maggots and wound healing: an investigation of the effects of secretions from Lucilia sericata larvae upon the migration of human dermal fibroblasts over a fibronectin-coated surface. Wound Repair Regen. 13(4):422-33.

14. LOUREIRO, M.; VANDERLEIA, C.; D'ALMEIDA, J. 2005. Desenvolvimento pós-embrionário de Pattonella intermutans (Thomson) (Diptera: Sarcophagidae) em diferentes dietas. Rev. Bras. Entomol. 49(1):127-129.

15. MENDOCA, P.; CARVALHO, M.; D'ALMEIDA, J. 2009. Rearing Chrysomya megacephala on artificial diets composed of varying concentrations of albumin. Braz. Arch. Biol. Technol. 52(1):421-426.

16. PAPE, T.; WOLFF, M.; AMAT, E. 2004. Los califóridos, éstridos, rinofóridos y sarcofágidos (Diptera: Calliphoridae, Oestridae, Rinophoridae, Sarcophagidae) de Colombia. Biota Colombiana. 5(2):201-208.

17. PARNÉS, A.; LAGAN, K. 2007. Larval therapy in wound management. Review. Int. J. Clin. Pract. 61(3): 488-493.

18. REY, M.; CASTAÑEDA, A.; GONZÁLEZ, J.; ACERO, V.; SEGURA, A.; ZAPATA, C.; GAONA, M.; RÍOS, D.; BELLO, F. 2008. Evaluación de la terapia larval en el proceso de curación de heridas infectadas con Pseudomonas aeruginosa en Conejos. Rev. Cienc. Salud. 6(2): 9-24.

19. RING, R. 1972. Relationship between diapause and supercooling in the blowfly, Lucilia sericata (Mg.) (Diptera: Calliphoridae). Can. J. Zool. 50(12):1601-1605.

20. RODRIGUERO, M.S.; VILARDI, J.C.; VERA, M.T.; CAYOL, J.P.; RIAL, E. 2002. Morphometric traits and sexual selection medfly (Diptera: Tephritidae) under field cage consditions. Florida Entomol. 85 (1):143-149.

21. SAIGUSA, K.; MATSUMASA, M.; YASHIMA, Y.; YOICHI.; AOKI, Y. 2009. Practical applications of molecular biological species identification of forensically important flies. Legal Medicine. 11:344-347.

22. SAUINDERS, D.; MACPHERSON, J.; CAIRNCROSS, K. 1986. Maternal and larval effects of photoperiod on the induction of larval diapause in two species of fly, Calliphora vicina and Lucilia sericata. Exp. Biol. 46(1): 51-58.

23. SHERMAN, R. 2002. Maggot therapy for foot and leg wounds. Lower Extremity Wounds. 1(2):135-142. 
24. SILVA, C.; MOYA, G.; AZAMBUJA, P. 2008. Use of polyester pad as a new physical substrate for rearing Cochliomyia hominivorax (Diptera: Calliphoridae) larvae. Neotrop. Entomol. 37(3):349-351.

25. Simple Interactive Statistical Analysis SISA Disponible desde internet en: http://www.quantitativeskills.com/ sisa/ (con acceso 29/07/10).

26. SOPER, D. 2010. Statictis Calculator, Analysis of Variance Calculator - One-Way ANOVA from Summary Data. Disponible desde Internet en: http://www.danielsoper. com/statcalc/calc43.aspx (con acceso 29/07/10).

27. TACHIBANA, S.; NUMATA, H. 2001. An artificial diet for blow fly larvae, Lucilia sericata (Meigen) (Diptera: Calliphoridae). Appl. Entomol. Zool. 36(4):521-523.

28. TENQUIST, J. 1971. Rearing of Lucilia sericata (Diptera:Calliphoridae) on a modified Musca domestica medium. N. Zeal. Entomol. 5: 30-31.
29. UJVARI, B.; WALLMAN, J.; MADSEN, T.; WHELAN, M.; HULBERT, A. 2009. Experimental studies of blowfly (Calliphora stygia) longevity: A little dietary fat is beneficial but too much is detrimental. Comparat. Biochem. Physiol.. 154(3): 383-388.

30. USAQUÉN, W.; CAMACHO, G. 2004. Ciclo de vida de Lucilia sericata (Diptera:Calliphoridae) como primera especie colonizadora presente en hígado humano realizado en el Instituto Nacional de Medicina Legal y Ciencias Forenses. Revista de INML y CF. 18(2):31-36.

31. VÉLEZ, M.; WOLFF, M. 2008. Rearing five species of Diptera (Calliphoridae) of forensic importance in Colombia in semicontrolled field conditions. Papéis Avulsos de Zoologia. 48(6):41-47.

32. ZHANG, B.; NUMATA, H.; MITSUI, H.; GOTO, S. 2009. A simple, heat-sterilizable artificial diet excluding animalderived ingredients for adult blowfly, Lucilia sericata. Med. Vet. Entomol. 23(4):443-447.

Recibido: Abril 21 de 2010

Aceptado: Agosto 2 de 2010 